\title{
Formulation of Decimation N Algorithm Expressions for Digital Signal Processor
}

\author{
Tae Ho Choi ${ }^{1}$ and Jun Ha Lee ${ }^{2}$ \\ ${ }^{1}$ Computer Information Telecommunication Engineering, Sangmyung University \\ ${ }^{2}$ Computer System Engineering, Sangmyung University \\ lascob@naver.com, ${ }^{2}$ junha@smu.ac.kr
}

In this article, expression of algorithm of Decimation $N$ of Multi-rate unning convolution algorithms operating filter output fast by processing Ramning Convolution filter with filter bank is reviewed according to each value of $n(n>-3)$ and then the part which is changed in regularly with value of $n$ is expressed as an algorithm.

Keywords: Decimation, Multi-rate running convolution algorithm, FFT (Fast Fourier Transform), DSP (Digital Signal Processgr)

\section{Introduction}

For fast algorithms reducing complexity of FIR filter operation, there are two methods: indirect method of operation (m frequencies including TTF (Fast Fourier Transformation) and direct method of reducing operation in time [1]. However, fast algorithms are not widely used because its hardware design requires pipeline structure which demands large area for the design and it is not effective in software design with DSP (Digital Signal Processor). That is, reduction in operations of these fast algorithms is the result of inefficient structure with low effectiveness $[2,37$. Nevertheress, if it can be designed effectively with process using MAC (Multiplication and Accumulation), reduction of operations will be significant. Accordingly, direct methods of fast aggorithms reducing operations of MAC have been developed $[4,5]$. And fast algorithm using pseudocirculant matrix with indirect method FFT was also presented.

In this article, expression of algorithm of Decimation $\mathrm{N}$ of Multi-rate running convolution algorithms operating filter output fast by processing Running Convolution filter with filter bank is reviewed according to each value of $n(n>=3)$ and then the part which is changed regularly with value of $\mathrm{n}$ is expressed as an algorithm.

\section{Deeimation Algorithm}

Generally, filter is expressed in frequencies as follows:

$$
Y(\mathrm{z})=H(\mathrm{z}) X(\mathrm{z})
$$

In this formula, $Y(\mathrm{z}), H(\mathrm{z}), X(\mathrm{z})$ are output signal $y[n]$, impulse response $h[n]$ and $\mathrm{z}$ transform of input signal $x[n]$, respectively[6-9].

\subsection{The Algorithm of Decimation 3}

Here, in case of $n=3$, it is decomposed with polyphase and then the subsequent formulas are reviewed. 


$$
\begin{aligned}
& X(z)=X_{0}\left(z^{3}\right)+z^{-1} X_{1}\left(z^{3}\right)+z^{-2} X_{2}\left(z^{3}\right) \\
& Y(z)=Y_{0}\left(z^{3}\right)+z^{-1} Y_{1}\left(z^{3}\right)+z^{-2} Y_{2}\left(z^{3}\right) \\
& H(z)=H_{0}\left(z^{3}\right)+z^{-1} H_{1}\left(z^{3}\right)+z^{-2} H_{2}\left(z^{3}\right)
\end{aligned}
$$

When the polyphase-decomposed formula (2) is substitute into the formula (1), the following relational expression in the $z$ is shown. Then, if expressed with omitting $\left(z^{3}\right)$ of the entire polynomial expressions, it is the formula (3) below.

$$
\begin{aligned}
Y_{0}+z^{-1} Y_{1}+z^{-2} Y_{2}= & {\left[H_{0} X_{0}+z^{-3} H_{2} X_{1}+z^{-3} H_{1} X_{2}\right] } \\
& +z^{-1}\left[H_{1} X_{0}+H_{0} X_{1}+z^{-3} H_{2} X_{2}\right] \\
& +z^{-2}\left[H_{2} X_{0}+H_{1} X_{1}+H_{0} X_{2}\right]
\end{aligned}
$$

This formula can be expressed with matrix like the formula (4) below.

$$
\left[\begin{array}{c}
Y_{0} \\
Y_{1} \\
Y_{2}
\end{array}\right]=\left[\begin{array}{rrc}
H_{0} & z^{-3} H_{2} & z^{-3} H_{1} \\
H_{1} & H_{0} & z^{-3} H_{2} \\
H_{2} & H_{1} & H_{0}
\end{array}\right]
$$

The right side of this formula is called the pseudocirculant matrix and it can be expressed with 9 sub-filters.

In order to reduce number of sub-filters used, $Y_{0}, Y_{1}$, and $Y_{2}$ of the formula (4) are processed respectively as ffllows:

$$
\begin{aligned}
Y_{0} & =H_{0} X_{0}+z^{-3} H_{2} X_{1}+z^{-3} H_{1} X_{2} \\
& \left.=H_{0} X_{0}-z^{-3} H_{1}-X_{1}-z^{-3} H_{2} X_{2}+z^{-3} H_{1}+H_{2}\right)\left(X_{1}+X_{2}\right) \\
Y_{1} & =H_{1} X_{0}-H_{0} X_{1}+z^{-3} H_{2} X_{2} \\
& \left.=-H_{0} X_{0}-H_{1} X_{1}+z^{-3} H_{2} X_{2}+{ }^{\prime} H_{0}+H_{1}\right)\left(X_{0}+X_{1}\right) \\
& =H_{2} X_{0}+H_{1} X_{1}+H_{0} X_{2} \\
& \left.\left.=-H_{0} X_{0}+H_{1} X_{1}-H_{2} X_{2}+{ }^{(} H_{0}+H_{2}\right){ }^{(} X_{0}+X_{2}\right)
\end{aligned}
$$

When processing with the formulas $(5 a),(5 b)$ and $(5 c)$, the formula can be calculated by dising 6 filters including $H_{0}, H_{1}, H_{2}, H_{0}+H_{1}, H_{1}+H_{2}, H_{0}+H_{2}$ and so on. If the given filter is a FIR filter with 60 tabs, it needs 60 multiplication operations. Of the formulas (5a), (5b) and (5c) are used, 6 different filters have each 20 tabs and 120 multiplication operations are required in total. However, one third of them are operated in low speed and 40 multiplication operations per sample are required [10,11]. Therefore, if the proposed algorithm is used, number of multiplication operations is reduced from 60 to 40. The formulas (5a), (5b) and (5c) can be expressed by using Toom-Cook Algorithm as follows [12]:

$$
\left[\begin{array}{l}
Y_{0} \\
Y_{1} \\
Y_{2}
\end{array}\right]=C_{3}\left\{A_{3}\left[\begin{array}{l}
H_{0} \\
H_{1} \\
H_{2}
\end{array}\right] * A_{3}\left[\begin{array}{c}
X_{0} \\
X_{1} \\
X_{2}
\end{array}\right]\right\}
$$


In this formula, $*$ stands for inner product and $A_{3}$ and $C_{3}$ are as follows, respectively:

$$
A_{3}=\left[\begin{array}{lll}
1 & 0 & 0 \\
0 & 1 & 0 \\
0 & 0 & 1 \\
1 & 1 & 0 \\
1 & 0 & 1 \\
0 & 1 & 1
\end{array}\right] \quad C_{3}=\left[\begin{array}{rrrrrr}
1 & -z^{-3} & -z^{-3} & 0 & 0 & z^{-3} \\
-1 & -1 & z^{-3} & 1 & 0 & 0 \\
-1 & 1 & -1 & 0 & 1 & 0
\end{array}\right]
$$

Number of rows in $A_{3}$ is that of sub-filters used and $C_{3}$ Stands for combination of sub-filters for expressing output.

The structure of filter by the formula (6) and (7) is like Figure 1(a)

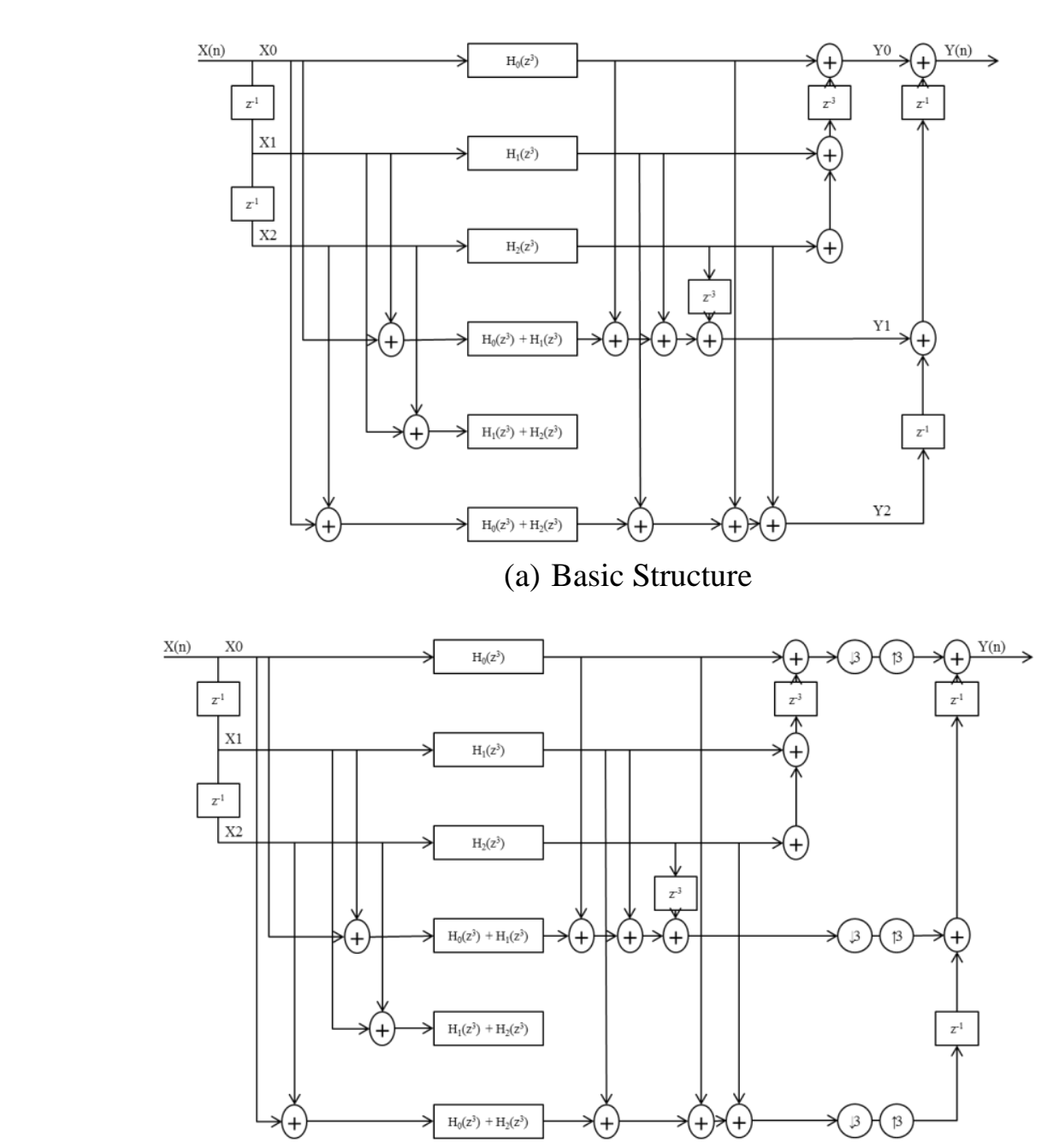

(b) Structure with inserting a sampler 


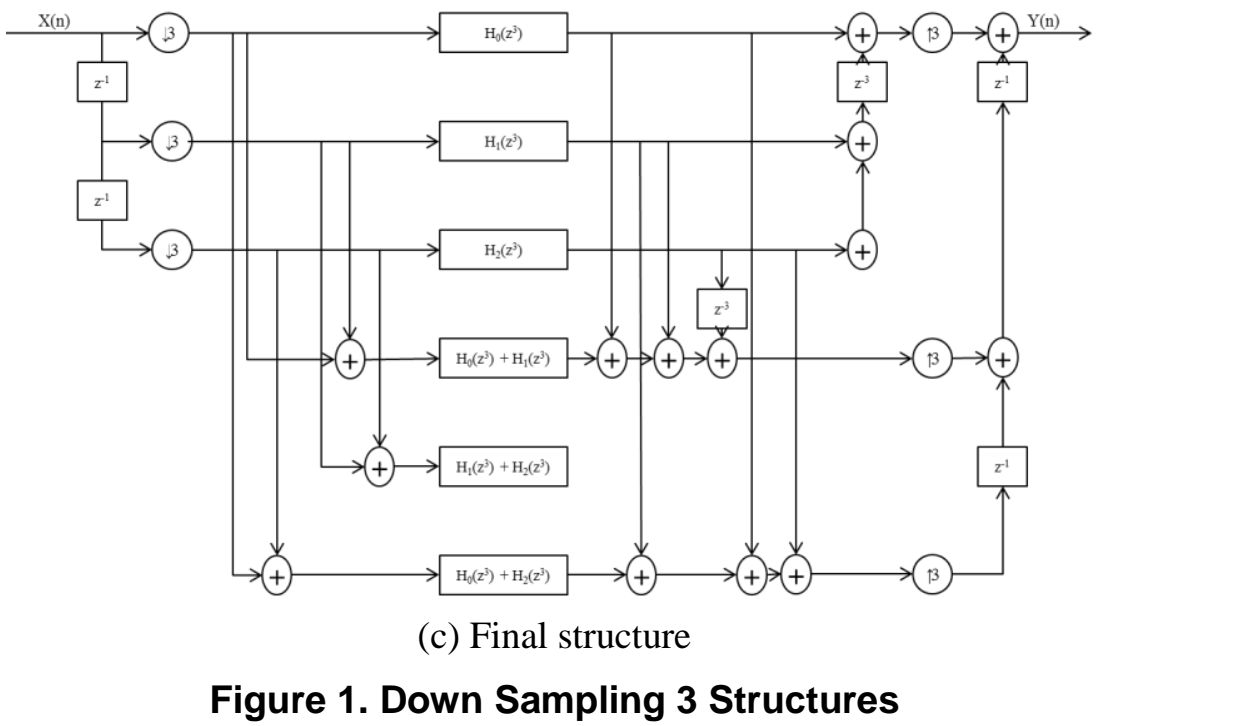

(a) Basic structure, (b) Structure with inserting a sampler, (c) Final structure

In Figure 1 (a), $Y_{0}\left(\mathrm{z}^{3}\right), Y_{1}\left(\mathrm{z}^{3}\right)$, and $Y_{2}\left(\mathrm{z}^{3}\right.$ are signals inserted with zero sample, down sampler 3 and up sampler 3 can be inserted like Figore $1^{\prime}$ (b). And in Figure 1 (b), filter of $\mathrm{z}^{-3}$ and down sampler 3 can be exchangeable in position by using Noble Identity and it can induce an effective structurelshown in the final Figure 1 (c). As seen in Figure 1 (c), inputted samples are reduced by one third of the speed by using down sampler and delay element and it is focused to delay element and up sampler one by one in the output.

The sub-filter $\tilde{A}_{3}$ except for matrix bilaterally symmetrical to the Identity matrix in $A_{3}$ of the formula (7) is as follows.
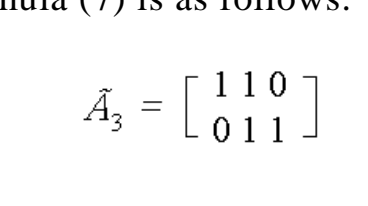

\subsection{The Algorithm of Decimation}

When the formula (1) is decomposed with polyphase of 4 , the relation of its input and output can be expressed in pseudocirculant matrix as follows:

$\left[\begin{array}{c}Y_{0} \\ Y_{1} \\ Y_{2} \\ Y_{3}\end{array}\right]=\left[\begin{array}{rrrr}H_{0} & z^{-4} H_{3} & z^{-4} H_{2} & z^{-4} H_{1} \\ H_{1} & H_{0} & z^{-4} H_{3} & z^{-4} H_{2} \\ H_{2} & H_{1} & H_{0} & z^{-4} H_{3} \\ H_{3} & H_{2} & H_{1} & H_{0}\end{array}\right]\left[\begin{array}{c}X_{0} \\ X_{1} \\ X_{2} \\ X_{3}\end{array}\right]$

In the formula (9), $Y_{0}, Y_{1}, Y_{2}$ and $Y_{3}$ are processed as follows:

$$
\begin{aligned}
& Y_{0}=H_{0} X_{0}+z^{-4} H_{3} X_{1}+z^{-4} H_{2} X_{2}+z^{-4} H_{1} X_{3} \\
& \left.\quad=H_{0} X_{0}-z^{-4} H_{1} X_{1}+z^{-4} H_{2} X_{2}-z^{-4} H_{3} X_{3}+z^{-4} H_{1}+H_{3}\right)\left(X_{1}+X_{3}\right) \\
& Y_{1}=H_{1} X_{0}+H_{0} X_{1}+z^{-4} H_{3} X_{2}+z^{-4} H_{2} X_{3}
\end{aligned}
$$




$$
\begin{aligned}
& \left.\left.=-H_{0} X_{0}-H_{1} X_{1}-z^{-4} H_{2} X_{2}-z^{-4} H_{3} X_{3}+{ }^{(} H_{0}+H_{1}\right)\left(X_{0}+X_{1}\right)+z^{-4}\left(H_{2}+H_{3}\right){ }^{(} X_{2}+X_{3}\right)(10 \mathrm{~b}) \\
Y_{2} & =H_{2} X_{0}+H_{1} X_{1}+H_{0} X_{2}+z^{-4} H_{3} X_{3} \\
& \left.\left.=-H_{0} X_{0}+H_{1} X_{1}-H_{2} X_{2}+z^{-4} H_{3} X_{3}+{ }^{(} H_{0}+H_{2}\right){ }^{(} X_{0}+X_{2}\right) \\
Y_{3} & =H_{3} X_{0}+H_{2} X_{1}+H_{1} X_{2}+H_{0} X_{3} \\
& \left.\left.\left.\left.=-H_{0} X_{0}-H_{1} X_{1}-H_{2} X_{2}-H_{3} X_{3}+H_{0}+H_{3}\right){ }^{(} X_{0}+X_{3}\right)+{ }^{(} H_{1}+H_{2}\right){ }^{(} X_{1}+X_{2}\right)
\end{aligned}
$$

The formula (10) can be expressed by using Toom-Cook Algorithm as follows:

$$
\left[\begin{array}{c}
Y_{0} \\
Y_{1} \\
Y_{2} \\
Y_{3}
\end{array}\right]=C_{4}\left\{A_{4}\left[\begin{array}{c}
H_{0} \\
H_{1} \\
H_{2} \\
H_{3}
\end{array}\right] * A_{4}\left[\begin{array}{c}
X_{0} \\
X_{1} \\
X_{2} \\
X_{3}
\end{array}\right]\right\}
$$

In this formula, $A_{4}$ and $C_{4}$ are as follows, respectively.

$$
A_{4}=\left[\begin{array}{llll}
1 & 0 & 0 & 0 \\
0 & 1 & 0 & 0 \\
0 & 0 & 1 & 0 \\
0 & 0 & 0 & 1 \\
1 & 1 & 0 & 0 \\
1 & 0 & 1 & 0 \\
1 & 0 & 0 & 1 \\
0 & 1 & 1 & 0 \\
0 & 1 & 0 & 1 \\
0 & 0 & 1 & 1
\end{array}\right]
$$

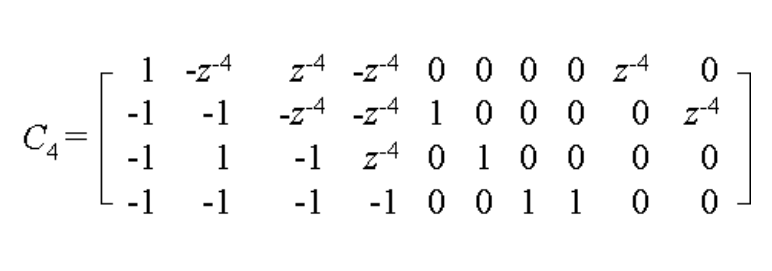

The sub-filter $\tilde{A}_{4}$ except for matrix bilaterally symmetrical to the Identity matrix in $A_{4}$ of the formula (12) is as follows:

\subsection{The Algorithm of Decimation 5}

When the formula (1) is decomposed with polyphase of 5, the relation of its input and output can be expressed in pseudocirculant matrix as follows:

$$
\left[\begin{array}{c}
Y_{0} \\
Y_{1} \\
Y_{2} \\
Y_{3} \\
Y_{4}
\end{array}\right]=\left[\begin{array}{rrrrr}
H_{0} & z^{-5} H_{4} & z^{-5} H_{3} & z^{-5} H_{2} & z^{-5} H_{1} \\
H_{1} & H_{0} & z^{-5} H_{4} & z^{-5} H_{3} & z^{-5} H_{2} \\
H_{2} & H_{1} & H_{0} & z^{-5} H_{4} & z^{-5} H_{3} \\
H_{3} & H_{2} & H_{1} & H_{0} & z^{-5} H_{4} \\
H_{4} & H_{3} & H_{2} & H_{1} & H_{0}
\end{array}\right]\left[\begin{array}{c}
X_{0} \\
X_{1} \\
X_{2} \\
X_{3} \\
X_{4}
\end{array}\right]
$$


In the formula (9), $Y_{0}, Y_{1}, Y_{2}, Y_{3}$ and $Y_{4}$ are processed as follows:

$$
\begin{aligned}
Y_{0}= & H_{0} X_{0}+z^{-5} H_{4} X_{1}+z^{-5} H_{3} X_{2}+z^{-5} H_{2} X_{3}+z^{-5} H_{1} X_{4} \\
= & H_{0} X_{0}-z^{-5} H_{1} X_{1}-z^{-5} H_{2} X_{2}-z^{-5} H_{3} X_{3}-z^{-5} H_{4} X_{4} \\
& \left.+z^{-5}\left(H_{1}+H_{4}\right)\left(X_{1}+X_{4}\right)+z^{-5} H_{2}+H_{3}\right)\left(X_{2}+X_{3}\right) \\
Y_{1}= & H_{1} X_{0}+H_{0} X_{1}+z^{-5} H_{4} X_{2}+z^{-5} H_{3} X_{3}+z^{-5} H_{2} X_{4} \\
= & -H_{0} X_{0}-H_{1} X_{1}-z^{-5} H_{2} X_{2}+z^{-5} H_{3} X_{3}-z^{-5} H_{4} X_{4} \\
& \left.\left.+\left(H_{0}+H_{1}\right)^{(} X_{0}+X_{1}\right)+z^{-5} H_{2}+H_{4}\right)\left(X_{2}+X_{4}\right) \\
Y_{2}= & H_{2} X_{0}+H_{1} X_{1}+H_{0} X_{2}+z^{-5} H_{4} X_{3}+z^{-5} H_{3} X \\
= & -H_{0} X_{0}+H_{1} X_{1}-H_{2} X_{2}-z^{-5} H_{3} X_{3}-z^{-5} H_{4} X_{4} \\
& +\left(H_{0}+H_{2}\right)\left(X_{0}+X_{2}\right)+z^{-5}\left(H_{3}+H_{4}\right)\left(X_{3}+X_{4}\right) \\
Y_{3}= & H_{3} X_{0}+H_{2} X_{1}+H_{1} X_{2}+H_{0} X_{3}+z^{-5} H_{4} X_{4} \\
= & -H_{0} X_{0}-H_{1} X_{1}-H_{2} X_{2}-H_{3} X_{3}+z^{-5} H_{4} X_{4} \\
& +\left(H_{0}+H_{3}\right)\left(X_{0}+X_{3}\right)+\left(H_{1}+H_{2}\right)\left(X_{1}+X_{2}\right) \\
& \\
Y_{4}= & H_{4} X_{0}+H_{3} X_{1}+H_{2} X_{2}+H_{1} X_{3}+H_{0} X_{4} \\
= & -H_{0} X_{0}-H_{1} X_{1}+H_{2} X_{2}-H_{3} X_{3}-H_{4} X_{4} \\
& +\left(H_{0}+H_{4}\right)\left(X_{0}+X_{4}\right)+\left(H_{1}+H_{3}\right)\left(X^{\prime}+X_{3}\right)
\end{aligned}
$$
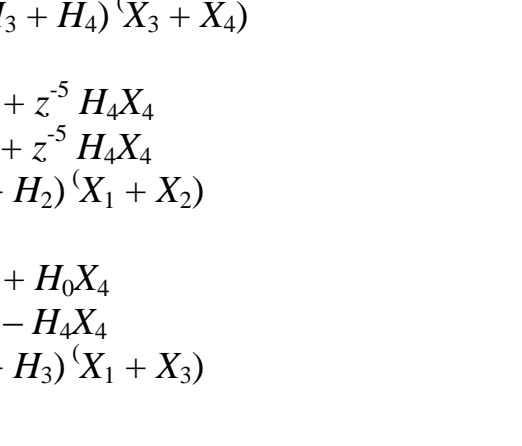

The formula (15) can be expressed by,using Toom-Cook Algorithm as follows:

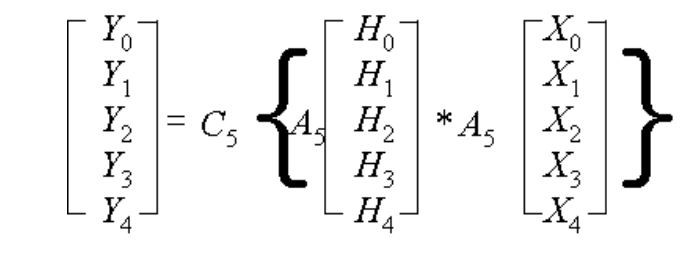

In this formula, $A_{5}$ and $\mathcal{C}_{5}$ are as follows, respectively:

$$
A_{5}=\left[\begin{array}{lllll}
1 & 0 & 0 & 0 & 0 \\
0 & 1 & 0 & 0 & 0 \\
0 & 0 & 1 & 0 & 0 \\
0 & 0 & 0 & 1 & 0 \\
0 & 0 & 0 & 0 & 1 \\
1 & 1 & 0 & 0 & 0 \\
1 & 0 & 1 & 0 & 0 \\
1 & 0 & 0 & 1 & 0 \\
1 & 0 & 0 & 0 & 1 \\
0 & 1 & 1 & 0 & 0 \\
0 & 1 & 0 & 1 & 0 \\
0 & 1 & 0 & 0 & 1 \\
0 & 0 & 1 & 1 & 0 \\
0 & 0 & 1 & 0 & 1 \\
0 & 0 & 0 & 1 & 1
\end{array}\right] C_{5}=\left[\begin{array}{rrrrrrrrrrrrrrrrr}
1 & -z^{-5} & -z^{-5} & -z^{-5} & -z^{-5} & 0 & 0 & 0 & 0 & 0 & 0 & z^{-4} & z^{-4} & 0 & 0 \\
-1 & -1 & -z^{-5} & 1 & -z^{-5} & 1 & 0 & 0 & 0 & 0 & 0 & 0 & 0 & z^{-4} & 0 \\
-1 & 1 & -1 & -z^{-5} & -z^{-5} & 0 & 1 & 0 & 0 & 0 & 0 & 0 & 0 & 0 & z^{-4} \\
-1 & -1 & -1 & -1 & z^{-5} & 0 & 0 & 1 & 0 & 1 & 0 & 0 & 0 & 0 & 0 \\
-1 & -1 & 1 & -1 & -1 & 0 & 0 & 0 & 1 & 0 & 1 & 0 & 0 & 0 & 0
\end{array}\right]
$$


The sub-filter $\tilde{A}_{5}$ except for matrix bilaterally symmetrical to the Identity matrix in $A_{5}$ of the formula (12) is as follows:

$$
\tilde{A_{5}}=\left[\begin{array}{lllll}
1 & 1 & 0 & 0 & 0 \\
1 & 0 & 1 & 0 & 0 \\
1 & 0 & 0 & 1 & 0 \\
0 & 1 & 1 & 0 & 0 \\
0 & 1 & 0 & 0 & 1 \\
0 & 0 & 1 & 1 & 0 \\
0 & 0 & 1 & 0 & 1 \\
0 & 0 & 0 & 1 & 1
\end{array}\right]
$$

\subsection{The Algorithm of Decimation $N$}

When the formula (1) is decomposed with polyphase of $\mathrm{N}$, the relation of its input and output can be expressed in pseudocirculant matrix as follows:

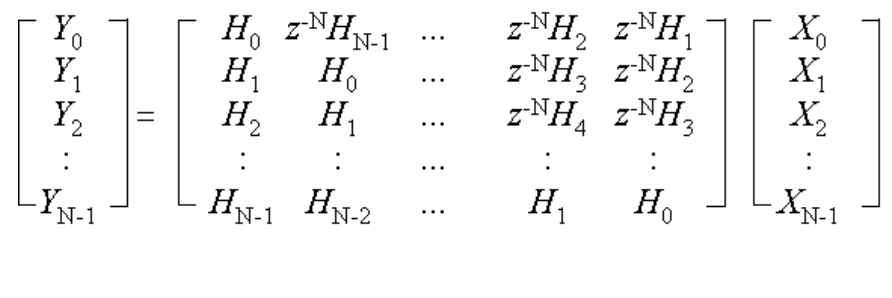

In the formula (9), in order to express $Y_{0}, Y_{1}, Y_{2}, Y_{3} \ldots$ and $Y_{\mathrm{N}-1}$, the following precedent expressions are required.

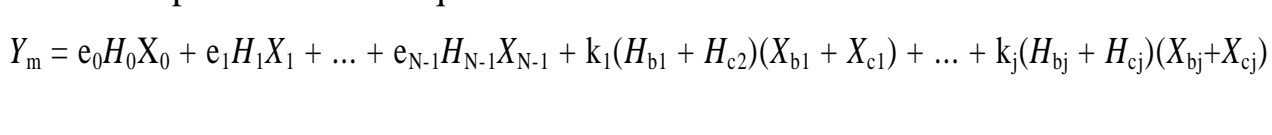

In the expression 1: $\left.\mathrm{kj} H \mathrm{bj}_{\mathrm{j}}+\mathrm{Hcj}\right)(\mathrm{Xbj}+\mathrm{Xcj})$, the range of value of $j$ is as follows:

If $\mathrm{N}$ is an odd number.

- $\mathrm{j}=(\mathrm{N}-1) /$

If $\mathrm{N}$ is anteven number,

- If ris an even number or $0, \mathrm{j}=\mathrm{N} / 2-1$

If $m$ is an odd number, $j=N / 2$

The expression 2; Of items in the formula of $\mathrm{Ym}$ (here, $\mathrm{m}$ is $0 \sim \mathrm{N}-1$ ), value of ea of eaHaXa(here, a is $0 \sim \mathrm{N}-1)$ can predict whether it is a positive number or a negative number. (But, in $\mathrm{kj}(\mathrm{Hbj}+\mathrm{Hcj})(\mathrm{Xbj}+\mathrm{Xcj})$, value of $k j$ is always plus)

In case $\mathrm{N}$ is an odd number,

- If $m$ is an even number or 0 , value of a is plus in case of $m / 2$ only and the rest is minus.

- If $m$ is an odd number, value of $a$ is $a$ is plus in case of $(\mathrm{N}-1) / 2+(m+1) / 2$ and the rest is minus. 
In case $\mathrm{N}$ is an even number,

- If $m$ is an even number or 0 , value of a is plus in two cases of $m / 2$ and $N / 2+m / 2$ and the rest is minus.

- If $\mathrm{m}$ is an odd number, value of a is always minus.

The expression 3: Of items in the formula $Y_{\mathrm{m}}($ here, $\mathrm{m}$ is $0 \sim \mathrm{N}-1)$, combination of $\mathrm{b}$ and $\mathrm{c}$ in the formula $\left(H_{\mathrm{b}}+H_{\mathrm{c}}\right)\left(X_{\mathrm{b}}+X_{\mathrm{c}}\right)($ here, $\mathrm{b}<\mathrm{c})$ is as follows:

Step 1: In case of $Y_{0}$,

- First, If ea is plus in the expression 1, and then it will be excluded.

- For b, starting from the smallest and for c, starting from the largest, the Combination is made.

- $\quad$ The formula $\mathrm{b}+\mathrm{c}=\mathrm{N}$ is made.

Step 2: In case of $Y_{\mathrm{m}}(0<\mathrm{m}<\mathrm{n})$

- First, if ea is plus in the expression 2, and then it will be exchuded.

- There is always the combination with-b=eand $c=m$.

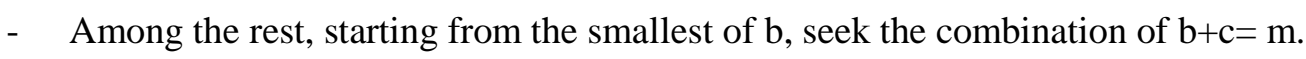

- If there are remaining yalues, among them, the smallest value is b. And seek combinations of $\mathrm{b}+\mathrm{c}=\mathrm{m}+\mathrm{N}$ and then every combination can be found.

The expression 4: Of items in the expression $Y_{\mathrm{m}}$ (here, $\mathrm{m}$ is $\left.0 \sim \mathrm{N}-1\right), z^{-\mathrm{N}}$ on values of $\mathrm{e}_{\mathrm{a}}$ and $\mathrm{k}_{\mathrm{j}}$ in the formulas e $H_{3} X_{\mathrm{a}}($ here, a $0 \sim \mathrm{N}-1)$ and $\mathrm{k}_{\mathrm{j}}\left(H_{\mathrm{b}}+H_{\mathrm{c}}\right)\left(X_{\mathrm{b}}+X_{\mathrm{c}}\right)($ here, $\mathrm{b}<\mathrm{c})$ respectively are as follows.

- $\quad \mathrm{e}_{\mathrm{a}} H_{\mathrm{a}} X_{\mathrm{a}}$ : If a $>\mathrm{m}, z^{-\mathrm{N}}$ exists in $\mathrm{a}$.

- $\quad \mathrm{k}_{\mathrm{j}}\left(H_{\mathrm{b}}+H_{\mathrm{c}}\left(X_{\mathrm{b}}+X_{\mathrm{c}}\right) \dot{\text { If }} \mathrm{b} 1>\mathrm{m}, z^{-\mathrm{N}}\right.$ exists in $\mathrm{k}_{\mathrm{j}}$

Example: Calculate a formula of $Y_{4}$ if $\mathrm{N}=7$.

For basic expressions, there are three expressions of $\left(H_{\mathrm{b}}+H_{\mathrm{c}}\right)\left(X_{\mathrm{b}}+X_{\mathrm{c}}\right)$ since $\mathrm{j}=(7$ 1) $/ 2=3$ according, to the expression 1 mentioned above.

Therefore, the expression of $Y_{4}$ can be expressed as follows:

$Y_{4}=\mathrm{e}_{0} H_{6} \mathrm{X}_{0}+\mathrm{e}_{1} H_{1} X_{1}+\mathrm{e}_{2} H_{2} X_{2}+\mathrm{e}_{3} H_{3} X_{3}+\mathrm{e}_{4} H_{4} X_{4}+\mathrm{e}_{5} H_{5} X_{5}+\mathrm{e}_{6} H_{6} X_{6}$

$+\mathrm{k}_{1}\left(H_{\mathrm{b} 1}+H_{\mathrm{c} 2}\right)\left(X_{\mathrm{b} 1}+X_{\mathrm{c} 1}\right)+\mathrm{k}_{2}\left(H_{\mathrm{b} 2}+H_{\mathrm{c} 2}\right)\left(X_{\mathrm{b} 2}+X_{\mathrm{c} 2}\right)+\mathrm{k}_{3}\left(H_{\mathrm{b} 3}+H_{\mathrm{c} 3}\right)\left(X_{\mathrm{b} 3}+X_{\mathrm{c} 3}\right)$

By applying the expression 2 to the formula a 1 , value of plus or minus signs of $\mathrm{e}_{0}, \mathrm{e}_{1}$, $\mathrm{e}_{2}, \mathrm{e}_{3}, \mathrm{e}_{4}, \mathrm{e}_{5}$, and $\mathrm{e}_{6}$, are calculated as follows:

$\begin{aligned} Y_{4}= & -\mathrm{e}_{0} H_{0} \mathrm{X}_{0}-\mathrm{e}_{1} H_{1} X_{1}+\mathrm{e}_{2} H_{2} X_{2}-\mathrm{e}_{3} H_{3} X_{3}-\mathrm{e}_{4} H_{4} X_{4}-\mathrm{e}_{5} H_{5} X_{5}-\mathrm{e}_{6} H_{6} X_{6} \\ & +\mathrm{k}_{1}\left(H_{\mathrm{b} 1}+H_{\mathrm{c} 2}\right)\left(X_{\mathrm{b} 1}+X_{\mathrm{c} 1}\right)+\mathrm{k}_{2}\left(H_{\mathrm{b} 2}+H_{\mathrm{c} 2}\right)\left(X_{\mathrm{b} 2}+X_{\mathrm{c} 2}\right)+\mathrm{k}_{3}\left(H_{\mathrm{b} 3}+H_{\mathrm{c} 3}\right)\left(X_{\mathrm{b} 3}+X_{\mathrm{c} 3}\right)\end{aligned}$

By applying the expression 3 to the formula a2, combinations of bj+cj in $\left(H_{\mathrm{bj}}+\right.$ $\left.H_{\mathrm{cj}}\right)\left(X_{\mathrm{bj}}+X_{\mathrm{cj}}\right)$ are calculated as follows:

$\begin{aligned} Y_{4}= & -\mathrm{e}_{0} H_{0} X_{0}-\mathrm{e}_{1} H_{1} X_{1}+\mathrm{e}_{2} H_{2} X_{2}-\mathrm{e}_{3} H_{3} X_{3}-\mathrm{e}_{4} H_{4} X_{4}-\mathrm{e}_{5} H_{5} X_{5}-\mathrm{e}_{6} H_{6} X_{6} \\ & +\mathrm{k}_{1}\left(H_{0}+H_{4}\right)\left(X_{0}+X_{4}\right)+\mathrm{k}_{2}\left(H_{1}+H_{3}\right)\left(X_{1}+X_{3}\right)+\mathrm{k}_{3}\left(H_{5}+H_{6}\right)\left(X_{5}+X_{6}\right)\end{aligned}$ 
By applying the expression 4 to the formula 3 , if values of $\mathrm{k}_{1} \sim \mathrm{k}_{3}$ are calculated, the final expression of $Y_{4}$ is as follows:

$$
\begin{aligned}
Y_{4}= & -H_{0} X_{0}-H_{1} X_{1}+H_{2} X_{2}-H_{3} X_{3}-H_{4} X_{4}-z^{-7} H_{5} X_{5}-z^{-7} H_{6} X_{6} \\
& +\left(H_{0}+H_{4}\right)\left(X_{0}+X_{4}\right)+\left(H_{1}+H_{3}\right)\left(X_{1}+X_{3}\right)+z^{-7}\left(H_{5}+H_{6}\right)\left(X_{5}+X_{6}\right)
\end{aligned}
$$

$\tilde{A}_{\mathrm{N}}$ except for matrix bilaterally symmetrical to the Identity matrix in $A_{\mathrm{N}}$ can be calculated as follows:

First, number of $\mathrm{N} x \mathrm{~m}$. the matrix $\mathrm{a}_{\mathrm{ik}}(\mathrm{i}=1,2,3, \ldots, \mathrm{m}$ and $\mathrm{k}=1,2,3, \ldots, \mathrm{N})$ is as follows:

$$
\begin{aligned}
& a_{11}: a_{12}: a_{13}: \ldots: a_{1 N} \\
& a_{21}: a_{22}: a_{23}: \ldots: a_{2 N} \\
& \ldots \ldots \ldots \ldots \ldots \ldots \ldots \ldots \ldots \ldots \ldots \ldots \ldots \ldots \ldots \\
& a_{m 1}: a_{m 2}: a_{m 3}: \ldots: a_{m N}
\end{aligned}
$$

Here, $\left.\mathrm{m}={ }_{\mathrm{N}} C_{2}-\mathrm{\llcorner N} / 2\right\lrcorner$

The condition is one row must contain two 1 and the rest of them have value of 0 .

1: $\quad$ Every value of the matrix is initiatized to 0

2: $\quad \mathrm{i}=1, \mathrm{k}=1$

3: $\mathrm{j}=\mathrm{k}$

4: $\quad$ if $(k+j)=N$ then goto 11

5: $\quad \mathrm{a}_{\mathrm{ik}}=1$

6: $\quad j=j+1$

7: $\quad a_{i j}=1$

8: $\quad$ if $\mathrm{j}=\mathrm{N}$ then goto 11

9: $\quad \mathrm{i}=\mathrm{i}+$

10: goto 4

11: $\mathrm{k}=\mathrm{k}+1$

12: $\mathrm{j}=\mathrm{k}$

13: if $\mathrm{k}=\mathrm{N}$ then end

14: goto 4

And in $A_{\mathrm{N}}$ values of $P_{\mathrm{N}}$ and $Q_{\mathrm{N}}$ can be divided. For value of row of the first 1 from eachrow in the $\tilde{A}_{\mathrm{N}}$,

- in ease $\mathrm{N}$ is an even number, if it is same or less than N/2, the row is $\mathrm{PN}$ and other case is $\mathrm{QN}$.

- in case $\mathrm{N}$ is an odd number, if it is same or less than $(\mathrm{N}+1) / 2$, the row is $P_{\mathrm{N}}$ and other case is $Q_{\mathrm{N}}$.

\section{Conclusion}

The expression of decimation $\mathrm{N}$ algorithm can be fixed as the expression mentioned above. Just there is difference in number of sub-filters in number of rows of $\tilde{A}_{\mathrm{N}}$ but there is no difference in number of rows of $\tilde{A}_{\mathrm{N}}$. It is difference resulted from processing the expression in $\tilde{A}_{\mathrm{N}}$. And there is difference in values of matrix in $\tilde{A}_{5}$ but it can change 
according to order of items in the expressions and there is no difference in the result. In case of using $\tilde{A}_{5}$ suggested here rather than that of from $\tilde{A}_{5}$, there is a constant regulation and values of $\tilde{A}_{\mathrm{N}}$ can be easily calculated through the algorithms suggested here without necessity of calculation of each expression.

\section{References}

[1] S. H. Shin, W. S. Kim and S. Choi, "Research of applied u-Health system using Inference Algorithm", Journal of the Korea Academia-Industrial cooperation Society, vol. 13, Issue 11, (2012), pp. 5518-5524, DOI: 10.5762/KAIS.2012.13.11.5518.

[2] M. Vetterli, "Running FIR and IIR filtering using multrate filer banks", IEEE Trans. Acoust., Speech, Signal Processing, vol. 36, (1988) May, pp. 730-738, DOI: 10.1109/29.1582.

[3] Z. Mou and P. Duhamel, "Short-length FIR filters and their use in fast nonrecursive filtering", IEEE Trans. Signal Processing, vol. 39, (1991) June, pp. 1322-1332, DOI: 10.1109/78.136539.

[4] M. Teixeira and D. Rodriguez, "A class of fast cyclic convglution algonthms based on block pseudocirculants", IEEE signal Processing Letters, vol. 2, (1995) May, ph. 92-94, DOI. 10.1109/97.386.287.

[5] S. k. Kwon and D. m. Lee, "Performance Analysis and Evaluation of Hybrid Compensation Algorithm for Localization", Journal of the Korea Academia-Industrial cooperation Society, vol 11, Issue 6, (2010), pp. 2263-2268, DOI: 10.5762/KAIS.2010.11.6.2263.

[6] S. 1. Oh and Y. b. Jang, "Low-power Heartbeat Detection Algorithm and Structure using Modified CIC Filter Banks", The Transactions of the Korean Institute of Electrical Engineers, vo,. 62, Issue 2, (2013), pp. 264-269, DOI: 10.5370/KIEE.2013.62.2.264.

[7] S. Kim, K. Lim and Y. Lee, "A Travel Time Fusion Algorithm Based on Point and Interval Detection Data", IJMUE, vol. 2, no. 4, (2007) October, pp. 67-84.DOI: 10.11099MUE.2007.60.

[8] H. -S. Park and D. -S. Ko, "Performance Elhancement of Wireless Datalink Modem using Channel Coding", IJMUE, vol. 6, no. 4, (2011) October pp.53-60.

[9] S. -W. Lee and S. -W. Kim, "Multi-Channel LED Dijer with Power Optimization Feedback Control Technique", IJMUE, vol. 7, no. 2, (2012) April,pp 183-188.

[10] H. j. shin and J. Ryu, "A Dynamic Hedging Strategy for Option Transaction Using Artificail Neural Networks", IJSEIA, vol. 6, no. 4. (2012) October, pp. 111-116.

[11] K. Sasai, J. Sveholm, G. Kitagata and T. Kinoshita, "A Practical Design and Implementation of Active Information Resource based Network Management System", IJEIC, vol. 2, Issue 4, (2011) November, pp. 6786.
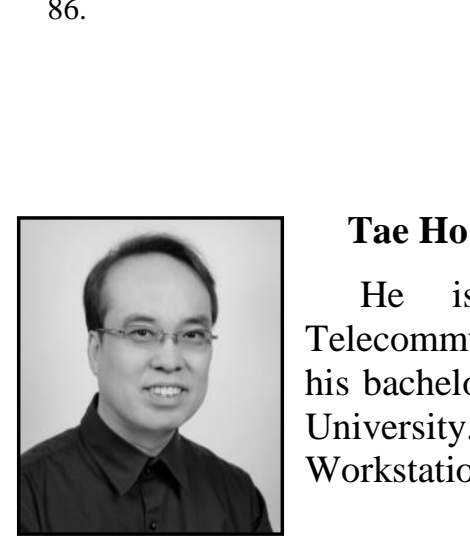

\section{Authors}

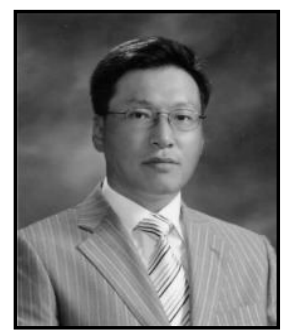

\section{Jun Ha Lee}

$\mathrm{He}$ is a Professor of Computer System Engineering at Sangmyung University. He received his Ph.D. from Chung-Ang. University in 1996. 